\title{
The atomic-scale microstructure of metal halide perovskite elucidated via low-dose electron microscopy
}

Mathias Rothmann ${ }^{1}$, Judy kim² ${ }^{2}$ Juliane Borchert ${ }^{3}$, Kilian Lohmann ${ }^{4}$, Colum O'Leary ${ }^{5}$, Alex Sheader ${ }^{6}$, Laura Clark ${ }^{7}$, Henry Snaith ${ }^{8}$, Michael Johnston ${ }^{8}$, Peter Nellist ${ }^{2}$ and Laura Herz ${ }^{8}$

${ }^{1}$ University of Oxford, Condensed Matter Physics, Oxford, England, United Kingdom, ${ }^{2}$ University of Oxford, Department of Materials, United States, ${ }^{3}$ University of Cambridge, United States, ${ }^{4}$ University of Oxford, Condensed Matter Physics, United States, ${ }^{5}$ University of California, Berkeley, United States, ${ }^{6}$ Department of Materials, University of Oxford, United States, ${ }^{7}$ University of Leeds, United States, ${ }^{8}$ Department of Physics, University of Oxford, United States

Understanding the atomic-scale crystallographic properties of photovoltaic semiconductor materials such as silicon, GaAs, and CdTe has been essential in their development from interesting materials to largescale energy conversion industries. However, studying photoactive hybrid perovskites by transmission electron microscopy (TEM) has proved particularly challenging due to the large electron energies typically employed in these studies.[1] In particular, the very close structural relationship between a number of crystallographic orientations of the pristine perovskite and lead iodide has resulted in severe ambiguity in the interpretation of EM-derived information, severely impeding the advance of atomic resolution understanding of the materials.

Here, we successfully image the archetypal $\mathrm{CH}\left(\mathrm{NH}_{2}\right)_{2} \mathrm{PbI}_{3}\left(\mathrm{FAPbI}_{3}\right)$ and $\mathrm{CH}_{3} \mathrm{NH}_{3} \mathrm{PbI}_{3}\left(\mathrm{MAPbI}_{3}\right)$ hybrid perovskites in their thin-film form with atomic resolution using a carefully developed protocol of lowdose STEM (see Figure 1).[2] Our images enable a wide range of previously undescribed phenomena to be observed, including a remarkably highly ordered atomic arrangement of sharp grain boundaries and coherent perovskite/ $\mathrm{PbI}_{2}$ interfaces, with a striking absence of long-range disorder in the crystal (see Figure 2). These findings explain why inter-grain interfaces are not necessarily detrimental to perovskite solar cell performance, in contrast to what is commonly observed for other polycrystalline semiconductors. Additionally, we observe aligned point defects and dislocations that we identify to be climb-dissociated, and confirm the room-temperature phase of $\mathrm{CH}\left(\mathrm{NH}_{2}\right)_{2} \mathrm{PbI}_{3}$ to be cubic. We further demonstrate that degradation of the perovskite under electron irradiation leads to an initial loss of $\mathrm{CH}\left(\mathrm{NH}_{2}\right)^{2+}$ ions, leaving behind a partially unoccupied, but structurally intact, perovskite lattice, explaining the unusual regenerative properties of partly degraded perovskite films. Our findings thus provide a significant shift in our atomic-level understanding of this technologically important class of lead-halide perovskites.

\section{Acknowledgements}

We thank the David Cockayne Centre for Electron Microscopy, University of Oxford, for access and support in the use of the JEOL ARM200F instrument (proposal number EP/K040375/1). We also thank Diamond Light Source for access and support in use of the electron Physical Science Imaging Centre (E02, MG21734) that contributed to the results presented here. L.M.H and M.B.J. thank the Humboldt Foundation for Research Awards. 
The authors gratefully acknowledge financial support from the Engineering and Physical Sciences Research Council (UK) (EPSRC) through grant number EP/P033229/1 and through the EPSRC CDT for New and Sustainable Photovoltaics.

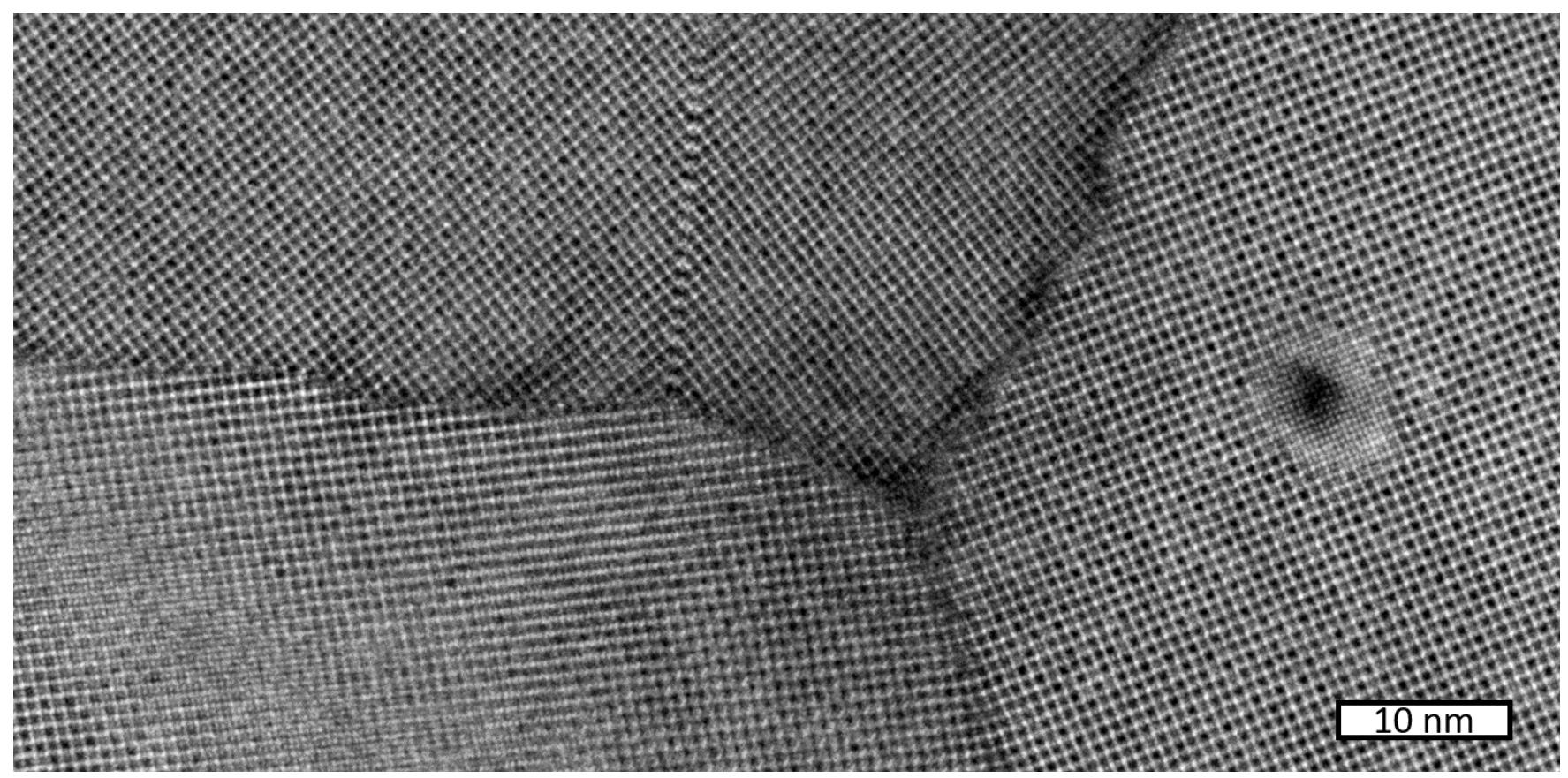

Figure 1. Low-angle annular dark-field scanning transmission electron micrograph illustrating several crystallographic phenomena of a FAPbI 3 thin film. Visible are high-angle and low-angle grain boundaries, a triple grain boundary, stacking faults, secondary phase inclusion, and a burned area containing the precursor PbI2 phase where the beam was parked for a brief moment. 


\section{A Partially FA-depleted perovskite}

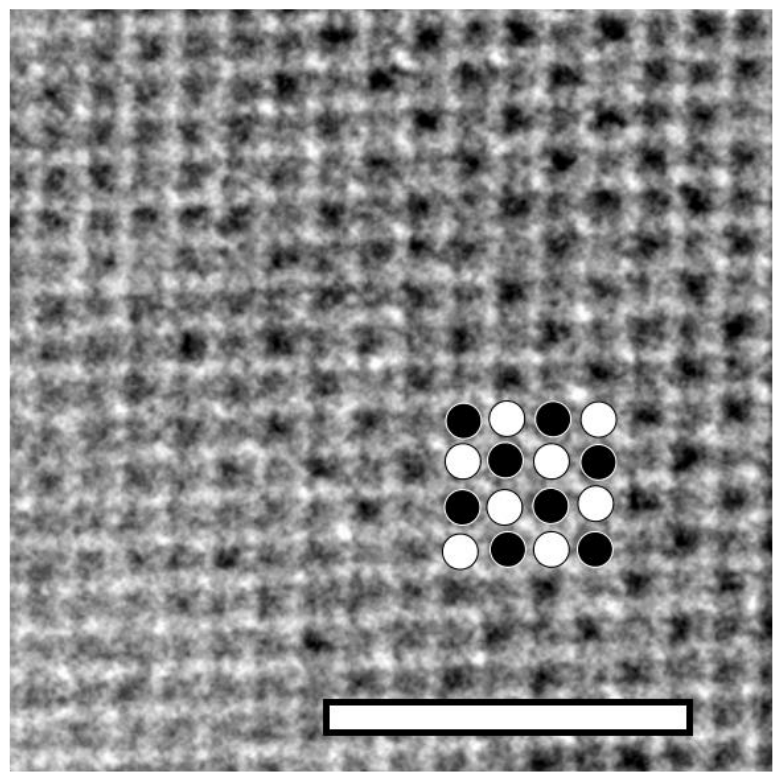

\section{Sharp $\mathrm{FAPbl}_{3}$ grain boundaries}

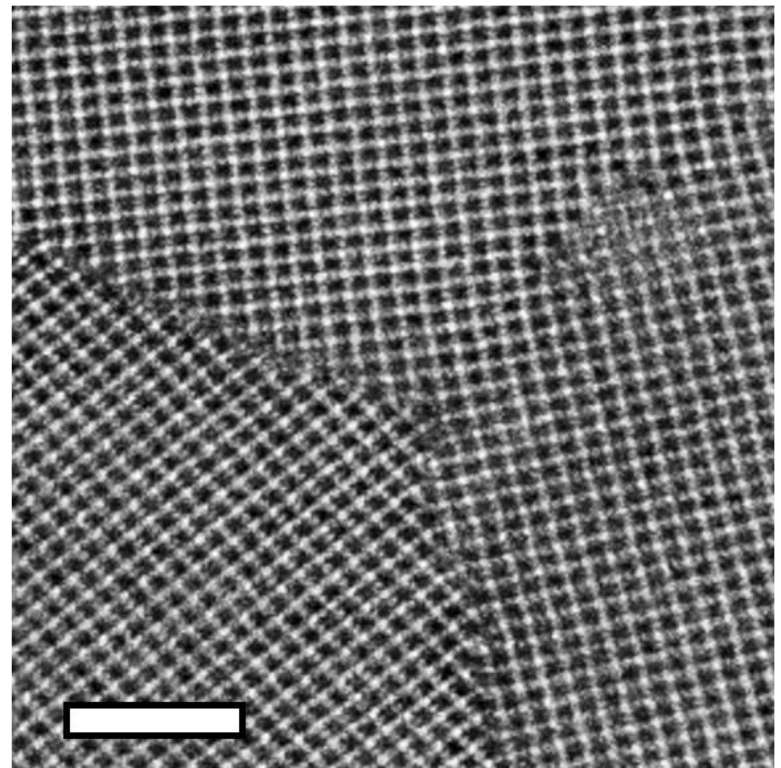

B Coherent $\mathrm{Pbl}_{2}: \mathrm{FAPbl}_{3}$ interface

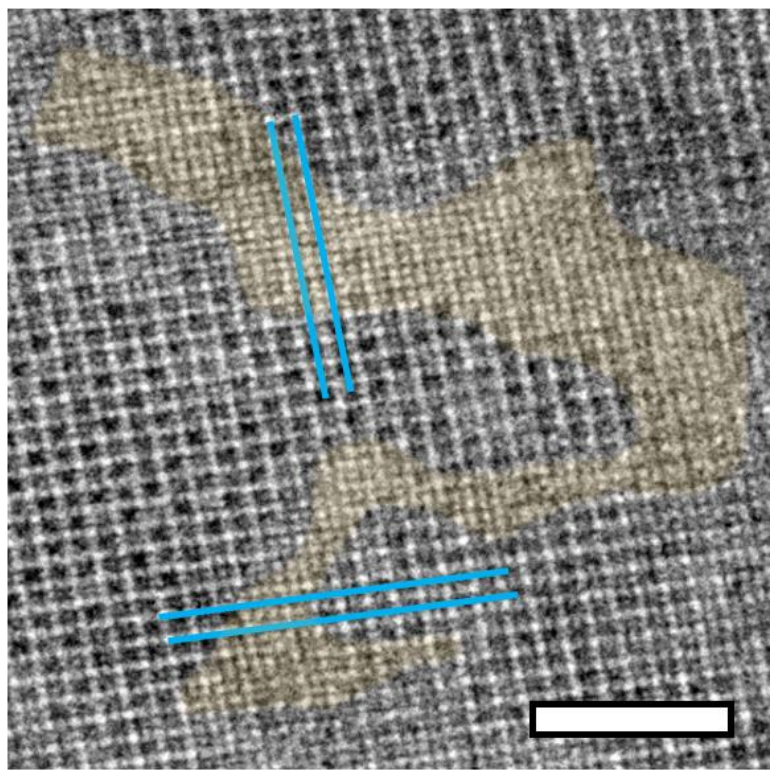

\section{Stacking fault and dislocation}

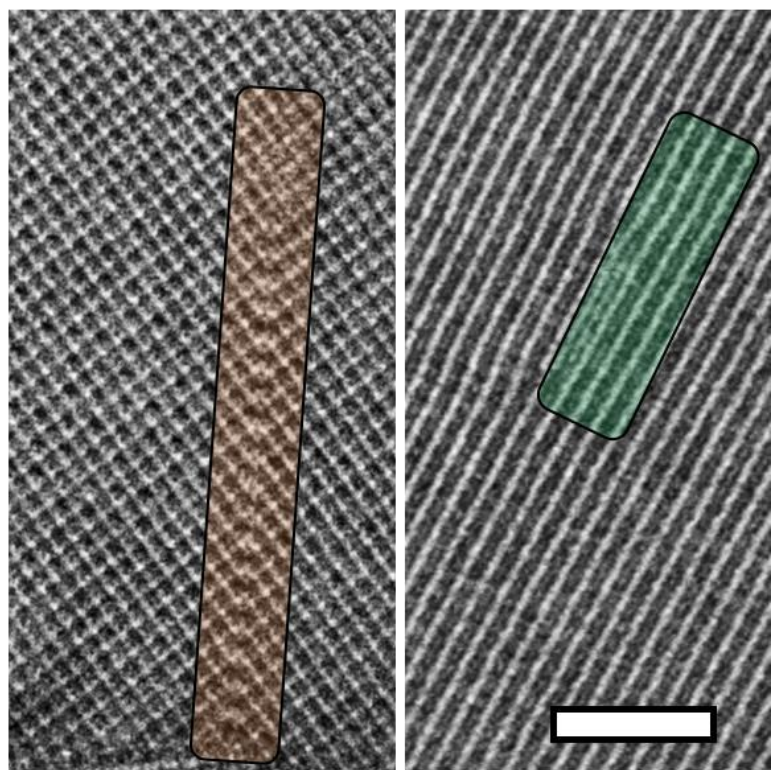

Figure 2. Overview of some key crystallographic properties of hybrid perovskite thin films including (A) a partially depleted crystal lattice after brief beam exposure, (B) highly coherent interfaces between FAPbI3 and PbI2, sharp grain boundaries with minimal distortions near the boundary, and (D) planar defects including stacking faults and dislocations.

\section{References}

[1] Adv. Mater. 2018, 30, 1800629

[2] Science 370, eabb5940 (2020) 